\title{
The Role of Confinement Loss in Highly Nonlinear Silica Holey Fibers
}

\author{
Vittoria Finazzi, Tanya M. Monro, and David J. Richardson
}

\begin{abstract}
Small-core holey fibers (HFs) can offer tight mode confinement, and are, therefore, attractive for highly nonlinear fiber applications. However, we show here that confinement loss can significantly degrade the performance of devices based on such small core fibers. We also identify a range of fiber designs that result in high fiber nonlinearity and low confinement loss. In particular, we show that pure silica HFs can exhibit effective nonlinearities more than 50 times higher than conventional fibers, and that the confinement loss can be lowered below the loss of standard fiber types.
\end{abstract}

Index Terms-Fiber design and fabrication, fiber properties, microstructured optical fibers, nonlinear optical fibers.

$\mathbf{H}$ OLEY FIBERS (HFs) are a class of microstructured optical fibers which guide light because the air holes that are present in the cladding region effectively reduce the refractive index relative to the solid core. HFs can have a significantly larger numerical aperture than conventional fiber types because the cladding region can be mostly comprised of air. When this is combined with a wavelength-scale core, HFs can provide tight mode confinement (i.e., small values of the effective mode area $\left.A_{\text {eff }}\right)$. In such fibers, high light intensities are guided within the core. Thus, even though silica is not intrinsically a highly nonlinear material [1], silica HFs can offer high effective fiber nonlinearities $\left(\gamma=(2 \pi / \lambda)\left(n_{2} / A_{\text {eff }}\right)\right.$, where $\lambda$ is the optical wavelength and $n_{2}$ is the nonlinear coefficient of the material [1]).

In the following, we will show that in pure silica HFs, the effective mode area can be as small as $1.7 \mu \mathrm{m}^{2}$ at $1550 \mathrm{~nm}$. Hence, assuming the $n_{2}$ of pure silica $\sim 2.2 \times 10^{-20} \mathrm{~m}^{2} / \mathrm{W}$ [1], nonlinearities as high as $\gamma \sim 52 \mathrm{~W}^{-1} \mathrm{~km}^{-1}$ are practical in these fibers, more than 50 times higher than in standard telecommunications fiber $\left(\gamma \sim 1 \mathrm{~W}^{-1} \mathrm{~km}^{-1}\right)$. Note that nonlinearities as high as $\gamma \sim 60 \pm 10 \mathrm{~W}^{-1} \mathrm{~km}^{-1}$ have been measured in silica HFs at $1550 \mathrm{~nm}$ [2]. This value is consistent with our theoretical prediction because of the uncertainty in the measurement of the value of $n_{2}$ for silica ( $n_{2} \approx 2.2-2.7 \times 10^{-20} \mathrm{~m}^{2} / \mathrm{W}[1]$ ). Using modified design conventional silica-based fibers, values of $\gamma$ have been limited to $20 \mathrm{~W}^{-1} \mathrm{~km}^{-1}$ thus far [3]. Hence, HFs offer enhanced nonlinearities relative to conventional fibers, and so are attractive for nonlinear fiber devices [4].

The loss in HFs occurs for a variety of reasons: intrinsic material absorption, additional losses arising during the fabrication process (water contamination, absorption due to impurities, scattering, etc.), and confinement loss [5]. In single-material

Manuscript received February 19, 2003; revised May 4, 2003.

The authors are with the Optoelectronics Research Centre, Southampton University, Southampton SO17 1BJ, U.K. (e-mail: vf@orc.soton.ac.uk).

Digital Object Identifier 10.1109/LPT.2003.816702
HFs, the core has the same refractive index as the material beyond the finite holey cladding region, and so every propagating mode is intrinsically leaky, and so experiences confinement loss [5]. Fabrication-related losses can be reduced by careful optimization, as shown recently in [6], which reports a loss as low as $0.58 \mathrm{~dB} / \mathrm{km}$ at $1550 \mathrm{~nm}$ for an $\mathrm{HF}$ with a hole-to-hole spacing $(\Lambda)$ of $4.2 \mu \mathrm{m}$. The losses so far measured in real HFs with smaller cores are significantly larger than this, and are typically of the order $50 \mathrm{~dB} / \mathrm{km}$ [7]. We have recently shown that confinement loss can contribute significantly to this increase in loss for small-core HFs [8].

In order to understand and reduce the impact of confinement loss, we have used the multipole method developed in [5] and [9] to analyze a variety of structures. The multipole method is a scattering technique that can be applied to any microstructured optical fiber with a cladding region defined by a finite number of circular inclusions of arbitrary refractive index. The approach is based on electric and magnetic field expansions valid in the vicinity of each hole. Here we consider air holes embedded in a uniform silica material. The cladding region is enclosed within a circular silica jacket with a complex refractive index, which allows the jacket to absorb the portion of the mode that leaks and, thus, the confinement loss to be estimated.

The multipole approach results in a homogeneous system of algebraic equations that contains just one unknown, the complex effective index $n_{\text {eff }}$ of the propagating mode. The problem can be formulated as a singular value problem, and the corresponding matrix scales in size with the number of air holes. Thus, the method is efficient and only becomes computationally intensive when many holes are considered [5]. By solving the matrix, the complex effective index $n_{\text {eff }}$ of the mode propagating in the HF can be found. Subsequently, the electric and magnetic vectorial fields of the propagating mode can be calculated, and the associated confinement loss can be predicted via the imaginary part of $n_{\text {eff }}$ [5].

We applied the multipole method to the study of small-core (highly nonlinear) HFs. The structures in the study contain between one and five rings of hexagonally packed holes. We consider fibers with hole-to-hole spacings in the range $0.8 \mu \mathrm{m}<$ $\Lambda<1.8 \mu \mathrm{m}$ and hole diameters in the range $0.6 \Lambda<d<$ $0.9 \Lambda$, and thus, the air-filling fraction (FF) lies between $33 \%$ and $74 \%\left(\mathrm{FF}=(\pi / 2 \sqrt{3})(d / \Lambda)^{2}\right)$. Fig. 1 shows two typical examples with different air-filling fractions but the same hole-to-hole spacing (named in the following Fibers I and II). The Poynting vector $S_{z}$ of the fundamental guided mode has been superimposed on each structure. The guided mode of Fiber I has an effective mode area of $\sim 3.2 \mu \mathrm{m}^{2}$, and is somewhat filamented in shape along the silica bridges. Increasing the air-filling fraction, 

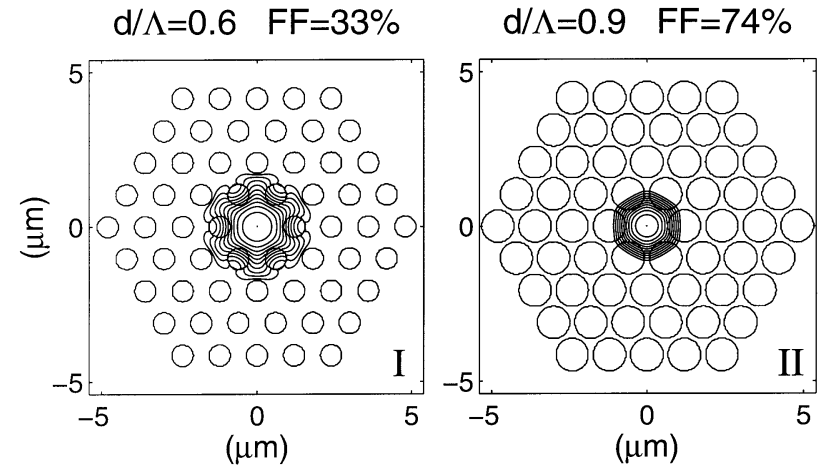

Fig. 1. Two typical structures considered in this study $(\Lambda=1.2 \mu \mathrm{m})$, labeled Fibers I and II. Contours represent the Poynting vector (i.e., transverse energy distribution) in decibels of the fundamental mode.

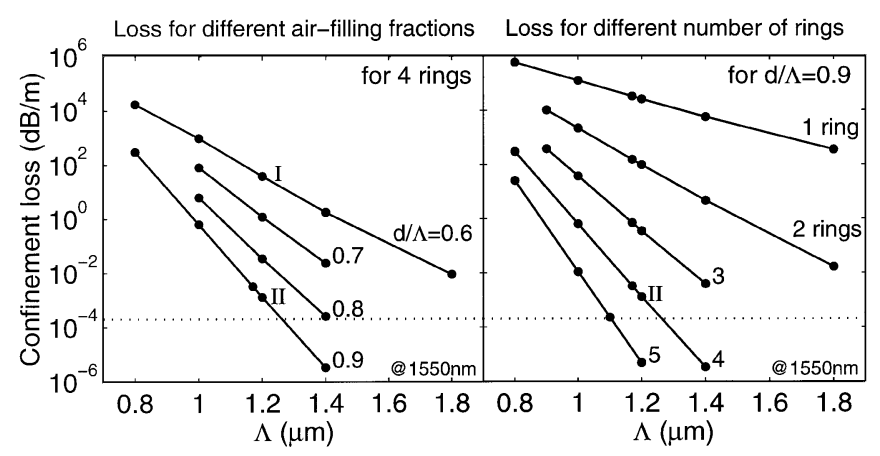

Fig. 2. Confinement loss for different air-filling fractions (left) and different number of rings of holes (right) as a function of $\Lambda$. The dotted line represents the loss of conventional fibers $(0.2 \mathrm{~dB} / \mathrm{km})$.

as in Fiber II, confines the mode more tightly to the core and, thus, reduces the effective mode area to $\sim 1.76 \mu \mathrm{m}^{2}$.

The graphs in Fig. 2 show the confinement loss as a function of the hole-to-hole spacing $(\Lambda)$ for a range of different HF structures. Each curve represents results for a given fiber profile scaled to a range of different dimensions. Observe that confinement loss always increases when the structure is reduced in scale. Consider now fibers with four rings of air holes, as in Fig. 2 (left). Using a bigger $d / \Lambda$ (i.e., larger air holes) reduces the loss for all values of $\Lambda$. This is unsurprising since the mode is always more tightly confined for larger air-filling fractions. Consider next fibers with a fixed air-filling fraction as in Fig. 2 (right). For all values of $\Lambda$, increasing the number of rings decreases the loss because the holey cladding extends over a larger region.

In previous works [5], HF structures with $\Lambda>\lambda$ were considered, and so the boundary of the cladding region was located relatively far from the core. However, we observe that when the $\mathrm{HF}$ is scaled to small dimensions, the degree of improvement that can be obtained by increasing the air-filling fraction [Fig. 2 (left)] or adding an extra ring of holes [Fig. 2 (right)] is limited. This occurs because the physical extent of the cladding region is reduced in the small scale structures considered in this study and, hence, the mode can leak out into the silica beyond the finite cladding structure. In addition, we have observed that reducing the dimension of the silica bridges does not reduce the confinement loss. Hence, in this regime, where the cladding features are subwavelength, the main loss contribution is due to the finite ex-

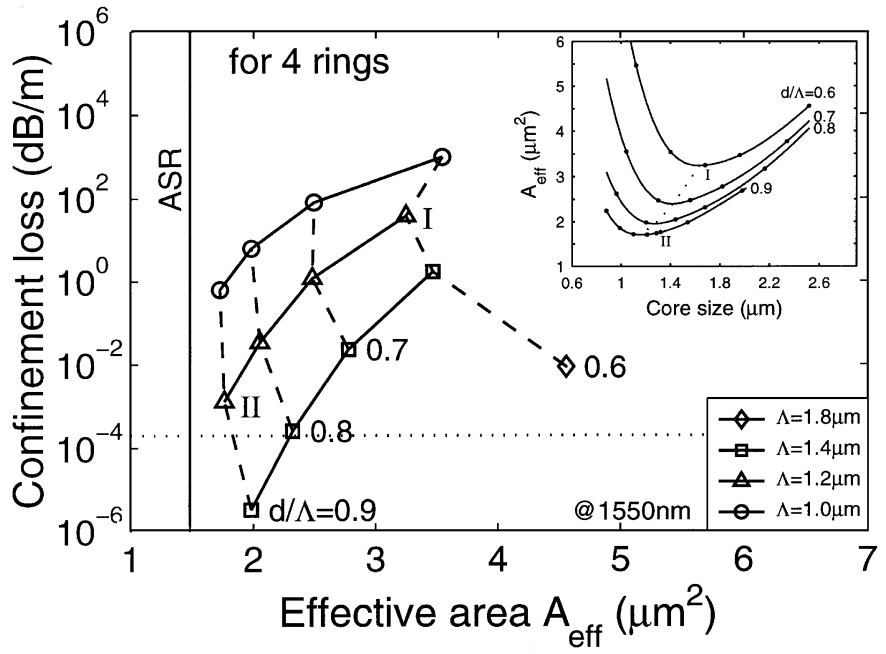

Fig. 3. Confinement loss versus effective mode area for a range of $\Lambda$ and $d / \Lambda$ with four rings. The solid vertical line represents the minimum mode area for the limit of a silica ASR. Inset: mode area for a range of HFs as a function of the core size $(2 \Lambda-d)$.

tent of the cladding structure, rather than leakage between the holes. Note that with careful design, the confinement loss can be reduced to values comparable with, or less than, the loss of conventional fibers $(0.2 \mathrm{~dB} / \mathrm{km})$, represented by the dotted line in Figs. 2 and 3.

The mode propagating in an HF interacts both with glass and the air in the holes, and this mode-air overlap can become significant when the core diameter is less than the wavelength of light. The nonlinear effects in HFs are induced by the portion of the field located in the glass since the nonlinearity of air is three order of magnitude smaller than that of silica. Hence, for all values quoted in this letter, the effective mode area $\left(A_{\text {eff }}\right)$ definition given in [1] is here modified as

$$
A_{\mathrm{eff}}=\frac{n_{2}\left[\iint E_{t}(x, y) \cdot E_{t}^{*}(x, y) d x d y\right]^{2}}{\iint \tilde{n}_{2}(x, y)\left[E_{t}(x, y) \cdot E_{t}^{*}(x, y)\right]^{2} d x d y}
$$

where $E_{t}(x, y)$ is the transverse electric field (* denotes the conjugate) and $\tilde{n}_{2}(x, y)$ is the nonlinear-index coefficient of the material at position $(x, y)$.

The inset of Fig. 3 shows the effective mode area as a function of the core size (defined as $2 \Lambda-d$ ) for a range of HFs with different air-filling fractions. As the diameter of the core is reduced, the mode becomes more confined. Once the core becomes significantly smaller than the optical wavelength, it is too small to confine the light well and the mode rapidly broadens again. Hence, for each curve there is a choice of $\Lambda$ that results in a minimum effective mode area. Observe that for smaller air-filling fractions, the minimum point is shifted to larger core dimensions (as shown by the dotted line), which reflects the fact that the index contrast between core and cladding is reduced. Unsurprisingly, the smallest effective mode area is achieved using the largest index contrast (i.e., when $d / \Lambda=0.9$ ). For this case, the minimum effective mode area value is $\sim 1.7 \mu \mathrm{m}^{2}$, only slightly larger than for extreme limit of an air-suspended rod (ASR), which is $\sim 1.48 \mu \mathrm{m}^{2}$. Note that, we find that the effective mode area remains remarkably constant regardless of the number of rings 
used. Essentially, although the tails of the mode significantly influence the mode's confinement loss, their impact on the effective mode area is minimal. Indeed, when just one ring of holes is considered, the effective mode area is only slightly larger than when two or more rings are present reflecting the fact that the mode is not so well confined.

Next, we explore whether it is possible to design fibers with small effective mode areas and reasonably low values of confinement loss, considering structures with four rings of holes only. The curves in Fig. 3 show the loss as a function of the effective mode area for four different values of the hole-to-hole spacing. The vertical line represents the theoretical minimum effective mode area that can be achieved in an air-suspended structure. For all hole-to-hole spacings, larger air-filling fractions not only reduce the loss but also decrease the effective mode area.

Observe from Fig. 3 that, regardless of the air-filling fraction, moving toward smaller core dimensions, there is a clear tradeoff between achieving small effective mode area and low confinement loss. Even though the hole-to-hole spacing $(\Lambda)$ can be chosen to minimize the value of the effective area of the fundamental mode $\left(A_{\text {eff }}\right)$, our loss calculations indicate that it is not always desirable to use the structures with the smallest effective mode area, because they typically exhibit higher confinement losses. A relatively modest increase in the structure scale in this small core regime can lead to dramatic improvements in the confinement of the mode without compromising the achievable effective nonlinearity significantly. Such graphs (Fig. 3) can be produced for a range of different cladding geometries, and they provide a useful practical design tool. Given an effective mode area required for a certain device and the magnitude of loss that can be tolerated (for a given device length), we can use this representation to identify the structures that minimize the fabrication difficulties (i.e., limit the number of rings).

We now concentrate on HF structures characterized by the air-filling fraction that produces the smallest effective area $(d / \Lambda=0.9)$, and analyze some device applications at $1550 \mathrm{~nm}$. Even in these idealized symmetric HFs, which are not birefringent, the polarization properties of these small-core fibers can significantly degrade device performance when the scale structure is subwavelength, but this is not discussed further here. We predict that Fiber II (introduced earlier) has an anomalous dispersion of $+14 \mathrm{ps} / \mathrm{nm} / \mathrm{km}$ and is, therefore, suitable for soliton-based devices. The confinement loss of Fiber II is calculated to be of order $\sim 1 \mathrm{~dB} / \mathrm{km}$, and the addition of another ring of holes reduces it below the loss level of conventional fibers. The high effective nonlinearity $\left(\gamma=45 \mathrm{~W}^{-1} \mathrm{~km}^{-1}\right)$ associated to this design allows a relatively short fiber length to be required for the nonlinear device, thus, the loss level of the practical four-ring design is perfectly tolerable.
Slightly reducing the hole-to-hole spacing of Fiber II to $1.17 \mu \mathrm{m}$, results in an HF with normal dispersion of $-14 \mathrm{ps} / \mathrm{nm} / \mathrm{km}$. This small normal dispersion is advantageous for optical thresholding and wavelength conversion devices because it reduces the impact of coherence degradation [10]. Note that the loss of this structure is still of the order of $\sim 1 \mathrm{~dB} / \mathrm{km}$, and its effective mode area is again approximately $1.75 \mu \mathrm{m}^{2}$. This example shows that it is, therefore, possible to tailor the dispersive properties of the fiber around the zero-dispersion wavelength without impacting other practical properties such as confinement loss and nonlinearity.

In conclusion, index-guiding pure-silica HFs can exhibit effective nonlinearities 50 times higher than conventional fibers. However, our simulations reveal that these small-core singlematerial fiber designs can suffer from significant confinement loss, a penalty that becomes more and more severe as the scale structure is reduced. Designs with large air-filling fractions and hole-to-hole spacings larger than $1 \mu \mathrm{m}$ are advantageous both in terms of achieving high nonlinearity and low confinement loss. In addition, near zero-dispersion at $1550 \mathrm{~nm}$ can be achieved. We have shown that it is possible to envisage HFs with $\gamma$ as high as $45 \mathrm{~W}^{-1} \mathrm{~km}^{-1}$ and reasonable confinement loss levels $(<0.2 \mathrm{~dB} / \mathrm{km})$ using just four rings of holes.

\section{REFERENCES}

[1] G. P. Agrawal, Nonlinear Fiber Optics. New York: Academic, 1989.

[2] W. Belardi, J. H. Lee, K. Furusawa, Z. Yusoff, P. Petropoulos, M. Ibsen, T. M. Monro, and D. J. Richardson, "A $10 \mathrm{Gbit} / \mathrm{s}$ tuneable wavelength converter based on four-wave mixing in highly nonlinear holey fiber," in Proc. ECOC, Copenaghen, Denmark, Sept. 2002, Paper PD1.2.

[3] T. Okuno, M. Onishi, T. Kashiwada, S. Ishikawa, and M. Nishimura, "Silica-based functional fibers with enhanced nonlinearity and their applications," IEEE J. Select. Topics Quantum., vol. 5, pp. 1385-1391, Sept./Oct. 1999.

[4] N. G. R. Broderick, T. M. Monro, P. J. Bennett, and D. J. Richardson, "Nonlinearity in holey optical fibers: Measurement and future opportunities," Opt. Lett., vol. 24, no. 20, pp. 1395-1397, Oct. 1999.

[5] T. P. White, R. C. McPhedran, C. M. de Sterke, L. C. Botten, and M. J. Steel, "Confinement losses in microstructured optical fibers," Opt. Lett., vol. 26, no. 21, pp. 1660-1662, Nov. 2001.

[6] L. Farr, J. C. Knight, B. J. Mangan, and P. J. Roberts, "Low loss photonic crystal fiber," in Proc. ECOC, Copenaghen, Denmark, Sept. 2002, Paper PD1.3.

[7] Z. Yusoff, J. H. Lee, W. Belardi, T. M. Monro, P. C. Teh, and D. J. Richardson, "Raman effects in a highly nonlinear holey fiber: Amplification and modulation," Opt. Lett., vol. 27, no. 6, pp. 424-426, Mar. 2002.

[8] V. Finazzi, T. M. Monro, and D. J. Richardson, "Confinement loss in highly nonlinear holey optical fibers," in Proc. OFC, OSA Tech. Dig., Anaheim, CA, Mar. 2002, pp. 524-525.

[9] T. P. White, R. C. McPhedran, L. C. Botten, G. H. Smith, and C. M. de Sterke, "Calculations of air-guided modes in photonic crystal fibers using the multipole method," Opt. Express, vol. 9, no. 13, pp. 721-732, Dec. 2001.

[10] N. Nakazawa, H. Kubota, and K. Tamura, "Random evolution and coherence degradation of a high-order optical soliton train in the presence of noise," Opt. Lett., vol. 24, no. 5, pp. 318-320, Mar. 1999. 